\title{
¿Qué indicadores son considerados por enfermería para conseguir una diálisis perfecta en el paciente en hemodiafiltración en línea?
}

\author{
"Premio Bellco al mejor trabajo de investigación sobre terapias convectivas $2011 "$
}

Ana Vanessa Fernández Martínez - Josefa Piñero Martínez - Yanina Arregui Arias - Rosario Pérez García - Alicia Moreno Vallejo - Francisco Rodríguez Martínez - Francisco José Orenes Bernabé - Matthew Mc Ginn

Enfermeras/os. Centro de diálisis Nephrocare FMC Service. Cartagena. Murcia

\section{Resumen}

Tanto las guías, como las indicaciones del Grupo de Gestión de Calidad de la Sociedad Española de Nefrología nos proponen una serie de indicadores para medir la calidad de la prestación de la hemodiálisis.

El objetivo del presente estudio fué, tras conocer cuales son los principales indicadores de una diálisis óptima para el personal de enfermería, evaluar objetivamente cada sesión de diálisis, en el convencimiento de que del conocimiento de los aciertos/fallos de cada sesión, obtendríamos propuestas de mejora de una forma más precoz que con los indicadores comúnmente utilizados.

Para ello, se realizó una encuesta entre el personal de enfermería $(n=26)$ solicitándole que enumerara los 10 parámetros que le parecían fundamentales para una sesión de diálisis óptima. Los 10 ítems más nombrados se utilizaron para elaborar la hoja de recogida de datos, con respuestas si/no.

Correspondencia:
Ana Vanessa Fernández
Centro de diálisis Nephrocare FMC Service
Paseo Alfonso XIII 61, 30203. Cartagena. Murcia
E-mail: nefroclubcarthago@gmail.com

En cada paciente $(n=160)$ se analizaron 3 sesiones consecutivas ( $n=480$ sesiones), con la misma pauta de tratamiento.

El enfermero que realiza las sesiones desconoce que sesiones se van a evaluar, así como la identidad de un segundo enfermero evaluador, que será el encargado de marcar si/no en cada uno de los ítems. Por cada objetivo conseguido se otorga 1 punto. La puntuación máxima por cada paciente es de 30 puntos (10 por sesión), excepto en los portadores de catéter tunelizado, en los que es de 27 puntos (9 por sesión), al no ser procedente valorar la hemostasia.

Hemos obtenido como resultado que el $41,3 \%$ de las sesiones realizadas obtuvieron la máxima puntuación (42,1\% con Fístula arteriovenosa y 35,1\% con catéter tunelizado, $p=0,043)$. La puntuación porcentual obtenida fue $88,7 \pm 11,9 \%$, porcentaje significativamente mayor con FAV que con catéter $(89,9 \pm 9,9$ versus $79,7 \pm 19,4, p<0,001)$. No se aprecian diferencias por sexo, edad, etiología 0 permanencia.

30 pacientes (18,8\%) obtienen la puntuación máxima en las tres sesiones. El 77,7\% de los pacientes (124) obtiene una puntuación superior al $85 \%$, y el $87,1 \%$ de los pacientes mayor del $80 \%$. 
Apenas en el $42,5 \%$ de los pacientes en las 3 sesiones se produce una completa recuperación del circuito limpia $(60,5 \%$ de las sesiones). Presentan síntomas durante la diálisis en al menos una sesión 50 pacientes (31,2\%), aunque solo el $15,2 \%$ de las sesiones son sintomáticas, lo que implica que existe una agrupación de síntomas en los mismos pacientes.

Todos los demás ítems se encuentran por encima del $90 \%$ de las sesiones, destacando el cumplimiento del Kt en un 96,3\% y del volumen de reinfusión en el $92,7 \%$, probablemente porque en el $92 \%$ de los casos se alcanza un flujo de sangre óptimo, y el tiempo prescrito se cumple en el $95 \%$ de las sesiones.

Por tanto, podemos concluir que la valoración objetiva en cada sesión de diálisis, en la intención de optimizarla, nos proporciona una valiosa información en tiempo real, y por tanto nos ofrece reafirmación en aquello que realizamos correctamente, y oportunidades de mejora en los aspectos que ejecutamos de forma más deficiente. La implicación de la enfermería nefrológica en este ciclo de mejora redundará de forma positiva en los clásicos indicadores de calidad que se miden de forma periódica, adelantando la solución antes de que se comunique el problema.

\section{PALABRAS CLAVE:}

- HEMODIAFILTRACIÓN EN LÍNEA

- ADECUACIÓN

- INDICADORES

What indicators are considered by nursing staff to achieve perfect dialysis in patients under on-line haemodiafiltration?

\section{Abstract}

Both the guides and the instructions of the Spanish nephrology society's quality management group offer a series of indicators for measuring quality in the provision of haemodialysis.

The aim of this study was, after identifying the main indicators of optimal dialysis in the view of nurses, to assess each dialysis session objectively, in the conviction that knowing what went right and wrong in each session would give us proposals for improvement, earlier than with the indicators in common use.

To this end, a survey was conducted among nursing staff $(n=26)$ asking them to list the 10 parameters they considered fundamental to a successful dialysis session. The 10 most-cited items were used to draw up the data gathering sheet, with yes/no responses.

For each patient $(n=160) 3$ consecutive sessions were assessed ( $n=480$ sessions), involving the same treatment pattern.

The nurse conducting the sessions was unaware which sessions were to be assessed, nor did they know the identity of a second assessing nurse, whose job would be to mark each of the items yes or no. 1 point was given for each objective achieved. The maximum score for each patient was 30 points (10 per session), except in those with tunnelled catheters, for whom it was 27 points (10 per session), as haemostasis is not assessed.

The result was that $41.3 \%$ of the sessions conducted got the top score $(42.1 \%$ with arteriovenous fistula and $35.1 \%$ with tunnelled catheter, $p=0.043$ ). The percentage score was $88.7 \pm 11.9 \%$, the percentage being significantly higher with AVF than with a catheter $(89.9 \pm 9.9$ versus $79.7 \pm 19.4, p<0.001)$. No differences by sex, age, aetiology or length of admission were detected.

30 patients ( $18.8 \%$ ) got the maximum score in all three sessions. El $77.7 \%$ of patients (124) scored over $85 \%$, and $87.1 \%$ of patients over $80 \%$.

In barely $42.5 \%$ was there a full recovery of the clean circuit in all three sessions ( $60.5 \%$ of the sessions). 50 patients $(31.2 \%)$ displayed symptoms during dialysis in at least one session, though only $15.2 \%$ of the sessions were symptomatic, implying that the symptoms were clustered in the same patients. 
All the other items were checked over $90 \%$ of the sessions, with KT compliance particularly high at $96.3 \%$ and reinfusion volume at $92.7 \%$, probably because in $92 \%$ of cases optimum blood flow was achieved and the prescribed time was achieved in $95 \%$ of sessions.

We can therefore conclude that objective assessment of each dialysis session, with the intention of optimising them, provides us with valuable real-time information, and therefore serves to confirm what we were doing right and highlight opportunities for improvement in aspects in which our performance is weaker. The involvement of nephrological nurses in this cycle of improvement will have a positive impact on the classic quality indicators which are regularly measured, anticipating the solution before the problem is reported.

\section{KEY WORDS:}

\section{- ON-LINE HAEMODIAFILTRATION \\ - SUITABILITY \\ - INDICATORS}

\section{Introducción}

Tanto las guías de la Sociedad Española de Nefrología (SEN) para centros de Hemodiálisis ${ }^{1}$, como la propuesta de indicadores del Grupo de Gestión de Calidad de la SEN ${ }^{2}$ nos proponen una serie de indicadores para medir la calidad de la prestación de la hemodiálisis. Así, nuestros resultados se comparan con el estándar obtenido del análisis bibliográfico o la opinión de los expertos. Estos indicadores se analizan con una frecuencia variable, pero siempre con una cadencia mayor de un mes.

\begin{tabular}{|c|c|c|}
\hline Item & Explicación & $\begin{array}{l}\text { Valoración } \\
\text { enfermera }\end{array}$ \\
\hline Acceso Vascular (AV) & $\begin{array}{l}\text { FAV: Sin dificultad de abordaje, sin necesidad } \\
\text { de recanalización, sin necesidad de volver a } \\
\text { puncionar } \\
\text { CT: Se retiran bien los tapones del sellado, no } \\
\text { signos de infección, no inversión de líneas }\end{array}$ & $73 \%(n=19)$ \\
\hline Flujo Sanguíneo (Qb) & $\begin{array}{l}\text { FAV: }>400 \mathrm{ml} / \mathrm{min} \text {, con límites de presiones } \\
\text { arterial y venosa } \pm 250 \text { y recirculación }<15 \% \text {; } \\
\mathrm{CT}:>350 \mathrm{ml} / \mathrm{min} \text { sin colapsos }\end{array}$ & $85 \%(n=22)$ \\
\hline Día Asintomática (DA) & & $81 \%(n=21)$ \\
\hline Estado de cámara y dializador D4C2 & $\begin{array}{l}\text { Completa recuperación del circuito sin } \\
\text { coagulaciones durante la sesión }\end{array}$ & $77 \%(n=20)$ \\
\hline Cumplimiento del tiempo de la sesión ( $T$ ) & $\begin{array}{l}\text { El tiempo efectivo no debe ser más de } \\
8 \text { minutos inferior al programado }\end{array}$ & $69 \%(n=18)$ \\
\hline Kt Óptimo & Ajustado a superficie corporal & $100 \%(n=26)$ \\
\hline Volumen de reinfusión on-line (VTR) $\geq 20$ litros & & $73 \%(n=19)$ \\
\hline Hemostasia en menos de 15 minutos (COA) & No resangrados & $77 \%(n=20)$ \\
\hline Buen estado paciente posdiálisis (BEG) & $\begin{array}{l}\text { Refiere encontrarse bien, constantes vitales } \\
\text { normales }\end{array}$ & $85 \%(n=22)$ \\
\hline El paciente alcanza su peso seco (PS) & Se admite una diferencia de $\pm 0,5 \mathrm{~kg}$. & $96 \%(n=25)$ \\
\hline
\end{tabular}

Tabla 1. Items y valoración enfermera 
Recientemente se ha comunicado como la implicación del personal de enfermería en cada sesión de hemodiálisis, puede mejorar los resultados de los indicadores en el área de adecuación de diálisis, cuando se mide el Kt por dialisancia iónica en tiempo real³.

El objetivo del presente estudio, tras conocer cuales son los principales indicadores de una diálisis óptima para el personal de enfermería, fue evaluar objetivamente cada sesión de diálisis, en el convencimiento de que del conocimiento de los aciertos/fallos de cada sesión obtendremos refuerzos y oportunidades de mejora de una forma más precoz que con los indicadores comúnmente utilizados.

\section{Pacientes y métodos}

Se realiza una encuesta entre el personal de enfermería $(n=26)$ solicitándole que enumere los 10 parámetros que le parecen fundamentales para una sesión de diálisis óptima. Los 10 ítems más nombrados se utilizan para elaborar la hoja de recogida de datos, con respuestas si/no. En la tabla 1 se recogen los ítems y el porcentaje de enfermeros que los sugieren.

Se realiza un estudio observacional sobre población prevalente en hemodiafiltración on-line (HDFOL) que presta su consentimiento, en dos centros de diálisis. En cada paciente $(n=160)$ se analizan 3 sesiones consecutivas ( $n=480$ sesiones).

Se reclutan 160 pacientes, $66 \%$ hombres, de 65,7 $\pm 14,9$ (27-87) años de edad, con una permanencia en diálisis de $56,5 \pm 59,5$ meses (1-324) y etiologías conocidas más frecuentes hipertensiva $(22,5 \%)$ y diabética $(22,5 \%) .141$ pacientes son portadores de fístula arteriovenosa (FAV) autóloga o protésica, mientras que 19 son portadores de catéteres tunelizados (CT). Todas las sesiones se realizan con helixona y helixone-HDF, siendo en las 3 sesiones analizadas idéntica la programación en cada paciente.

El enfermero que realiza las sesiones desconoce que sesiones se van a evaluar, así como la identidad de un segundo enfermero evaluador, que será el encargado de marcar si/no en cada uno de los ítems. Por cada objetivo conseguido se otorga 1 punto. La puntuación máxima por cada paciente es de 30 puntos (10 por sesión), excepto en los portadores de CT, en los que es de 27 puntos ( 9 por sesión), al no ser procedente valorar la hemostasia. Cada hoja de registro es firmada por el enfermero evaluador, indicando las fechas pertinentes de las tres sesiones de diálisis.

El análisis estadístico se realiza con el programa SPSS 13.0 para Windows. Las variables descriptivas cuantitativas se expresan como media, desviación estándar y rango. Las variables cualitativas, como frecuencias y porcentajes. El contraste de variables se realiza mediante t-student, y ANOVA para variables cuantitativas, y chi-cuadrado para variables cualitativas. Se acepta una significación estadística para $p<0,05$ ).

\section{Resultados}

El $41,3 \%$ de las sesiones realizadas obtuvieron la máxima puntuación $(42,1 \%$ con $\mathrm{FAV}$ y $35,1 \%$ con $C T, p=0,043)$. La puntuación porcentual obtenida fue $88,7 \pm 11,9 \%$, porcentaje significativamente mayor con FAV que con catéter $(89,9 \pm 9,9$ versus $79,7 \pm$ $19,4, p<0,001$ ). No se aprecian diferencias por sexo, edad, etiología o permanencia.

30 pacientes ( $18,8 \%)$ obtienen la puntuación máxima en las tres sesiones. El 77,7\% de los pacientes (124) obtiene una puntuación superior al $85 \%$, y el $87,1 \%$ de los pacientes mayor del $80 \%$.

Los datos obtenidos por paciente y sesión se observan en los gráficos 1 y 2 .

Apenas en el $42,5 \%$ de los pacientes en las 3 sesiones se produce una completa recuperación del circuito limpia (60,5\% de las sesiones). Presentan síntomas durante la diálisis en al menos una sesión 50 pacientes $(31,2 \%)$, aunque solo el $15,2 \%$ de las sesiones son sintomáticas, lo que implica que existe una agrupación de síntomas en los mismos pacientes.

Todos los demás ítems se encuentran rondando o por encima del $90 \%$ de las sesiones, destacando el cumplimiento del Kt en un $96,3 \%$ y del VTR en el $92,7 \%$, probablemente porque en el $92 \%$ de los casos se alcanza un Qb óptimo, y el tiempo prescrito se cumple en el $95 \%$ de las sesiones.

Mención especial cabe para el peso seco que fue alcanzado en el $89,4 \%$ de las sesiones, teniendo en 
cuenta que $1 / 3$ parte de las mismas corresponden a la primera sesión de la semana, tras el periodo inter dialítico más largo.

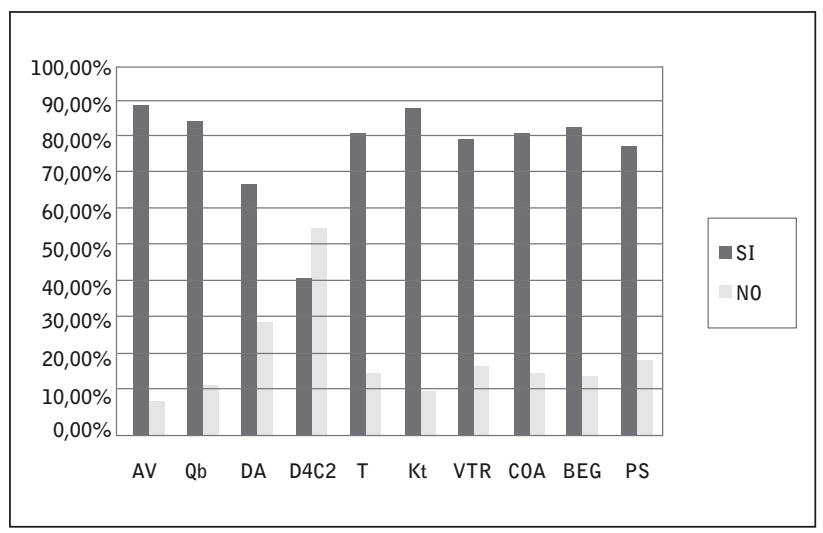

Gráfico 1. Resultados de ítems por paciente (referido a las 3 sesiones)

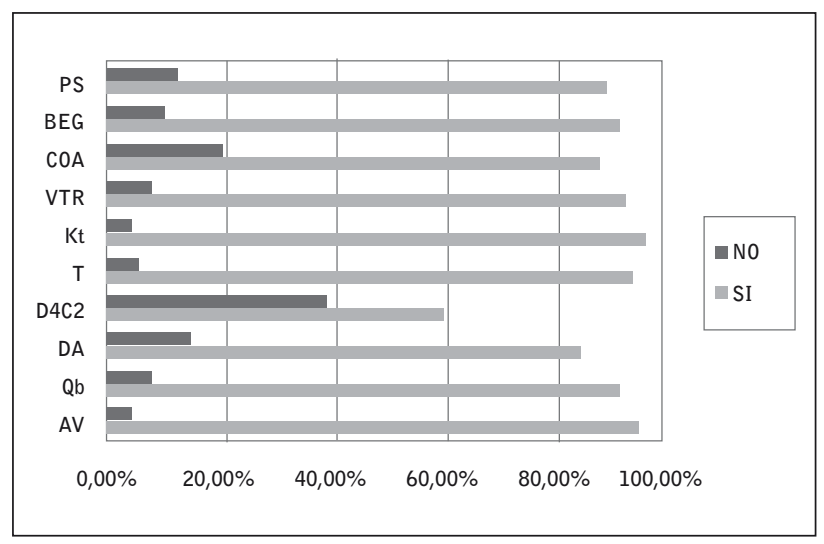

Gráfico 2. Resultados de ítems por sesión

\section{Discusión}

La magnitud de la convección se ha descrito como esencial en los mejores resultados de la HDFOL en comparación con otras técnicas de hemodiafiltración y hemodiálisis, y posiblemente puede tener relación con la supervivencia del paciente ${ }^{4-7}$. Por otro lado, la dosis de diálisis es considerada como un elemento fundamental en la diálisis adecuada y en la supervivencia del paciente ${ }^{8}$. La medición por Kt de la dosis de diálisis ha sido comunicada como más eficaz que mediante el Kt/V ${ }^{9}$, ya que este infraestima los casos de diálisis inadecuada, requiere determinaciones analíticas, es manipulable y no medible en cada sesión. Tanto Kt como VTR se influyen de forma sustancial por el flujo sanguíneo y el tiempo efectivo de la sesión. Nuestros resultados son congruentes, ya que en el $92 \%$ de las sesiones obtenemos un Qb óptimo y en el $95 \%$ alcanzamos el tiempo efectivo, por lo que el Kt y el VTR fijados se alcanzan en más del $90 \%$ de las sesiones.

El tiempo efectivo de la sesión se suele ver reducido con los monitores de diálisis más modernos, que interrumpen la diálisis para efectuar las mediciones pertinentes y con las alarmas ${ }^{10}$. Esto debería ser contemplado en la prescripción y ser lo más eficientes que sea posible, como en nuestro estudio en el que alcanzamos el objetivo en el $95 \%$ de los casos.

La hemodiálisis con catéter venoso central presenta una eficacia reducida con respecto a la realizada con fístula arteriovenosa, por lo que en muchos casos se precisa incrementar la duración de la sesión ${ }^{11}$. Sin embargo, cuando el flujo sanguíneo es adecuado, es posible alcanzar los objetivos deseados, incluso en hemodiafiltración on-line ${ }^{12}$. En cualquier caso, y pese a los esfuerzos de optimización, en nuestro estudio los pacientes portadores de catéter tunelizado tienen peores resultados que el conjunto.

El estado de hidratación en el paciente en hemodiálisis es de singular importancia, habiéndose descrito complicaciones derivadas del incremento del volumen extracelular (HTA, hipervolemia y disfunción cardiaca), o del descenso del mismo (hipotensión y mala tolerancia a la diálisis) ${ }^{13}$. El hecho de que el $15 \%$ de las sesiones evaluadas son sintomáticas, nos proporciona una oportunidad de mejora, por lo que hemos incorporado, tal como se describe en la bibliografía ${ }^{14}$, de forma sistemática la bioimpedancia multifrecuencia en el cálculo del peso seco, y recuperado algunas viejas estrategias en la prevención de la hipotensión intradiálisis ${ }^{15}$.

El apartado de nuestro estudio con resultados más decepcionantes es el de la recuperación limpia del circuito, apenas conseguido en el $60,5 \%$ de las sesiones. Probablemente estos resultados pueden estar interferidos por la reciente sustitución de la heparina convencional por la de bajo peso molecular en nuestros centros. En cualquier caso, también hemos modificado el protocolo de desconexión aumentando el volumen de infusión con el objetivo de mejorar este parámetro. 


\section{Conclusiones}

La valoración objetiva de cada sesión de diálisis en la intención de optimizarla nos proporciona una valiosa información en tiempo real, y por tanto nos ofrece reafirmación en aquello que realizamos más que correctamente, y oportunidades de mejora en los aspectos que ejecutamos de forma más deficiente. La implicación de la enfermería nefrológica en este ciclo de mejora redundará de forma positiva en los clásicos indicadores de calidad que se miden de forma periódica, adelantando la solución antes de que se comunique el problema.

Recibido: 20 Noviembre 2011

Revisado: 30 Noviembre 2011

Modificado: 10 Enero 2012

Aceptado: 20 Enero 2012

\section{Bibliografía}

1. Angoso M, Alcalde G, Álvarez-Ude F y Arenas MD. Guías SEN de centros de hemodiálisis. Gestión de la calidad en hemodiálisis. Nefrología 26 (Supl. 8): 73-87, 2006.

2. López Revuelta K, Barril G, Caramelo C, y cols: Desarrollo de un sistema de monitorización clínica para hemodiálisis: propuesta de indicadores del Grupo de Gestión de Calidad de la SEN. Nefrología 27: 542-559, 2007.

3. Fernández $A V$, Pereira $M^{a} S$, Vilar $M^{a} \mathrm{~V}$ y cols. Kt como indicador de dosis adecuada en una unidad de hemodiálisis: Estudio prospectivo. Libro de comunicaciones presentadas al XXXIV Congreso Nacional de la SEDEN. Pamplona 2009.

4. Maduell F. Convección versus difusión. ¿Ha llegado el momento del cambio? Nefrología 2009; 29(6):589-593.

5. Alfaro A, Beltrán MI, Gallego B y cols. HDF en línea en nuestros pacientes: calidad de vida y capacidad funcional. Premio Fresenius Medical Care he-

modiafiltración on-line. Rev Soc Esp Enferm Nefrol 2006; 9 (3) 158-163.

6. Canaud B, Braga-Gresham JL, Marshal MR y cols. Mortality risk for patients receiving haemodiafiltration versus haemodialysis: European results from the DOPPS. Kidney Int. 2006; 69: 2087-2093.

7. Jirka T, Cesare S, Di Benedetto y cols. Mortality risk for patients receiving hemodiafiltration versus hemodialysis. Kidney Int 2006, 70, 1524.

8. Held PJ, Port FK, Wolfe RA, Stannard DC, y cols: The dose of hemodialysis and patients mortality. Kidney Int 1996; 50: 550-556,

9. Fernández AV, Soto $S$, Arenas M, y cols. Estudio comparative de la dosis de diálisis medida por Kt y Kt/V. Rev Soc Esp Enferm Nefrol 2009;12(2):97-102.

10. Molina M, Roca S, de Alarcón RM y cols. Nefrología 2010;30(3);331-336

11. Maduell F, Vera M, Arias $M$, y cols. ¿Cuánto tiempo es necesario aumentar la prescripción de hemodiálisis con la utilización de catéteres? Nefrología 2008; 28:577-580.

12. Maduell F, Blasco M, Vera $M$, y cols,. Hemodiafiltración on-line con catéter venoso central. Nefrología 2007; 27 (Supl 4): 57,

13. Lopez Gomez JM, Jofré R. Balance hidrosalino en pacientes en hemodiálisis y su repercusión cardiovascular. Jofré R, Lopez Gomez JM, Luño J, Perez García R, Rodriguez Benitez P. En: Tratado de hemodiálisis. $2^{a}$ Edic Medica JIMS, Barcelona; 436440. 2006.

14. Fernández AV, Cegarra $R$, Aznar $S$ y cols. Estimación del peso seco en el paciente en hemodiálisis: ¿Coincidimos todos? Libro de Comunicaciones Presentadas al XXXV Congreso Nacional SEDEN. 2010 Granada.

15. Alfaro A, Gallego BE, Martín M y cols. Prevención de hipotensiones intradiálisis. Estudio comparativo: perfiles de sodio frente a hipotermia. Libro de comunicaciones presentadas al XVIII Congreso Nacional de la SEDEN. 2003. 